\title{
Preoperative Ca 19-9 Serum Levels as a Predictive Biomarker of Resecta Bility in Pancreatic Adenocarcinomas
}

Khalid Mazine*, Abdesslam Bouassria, Khalid Ait Taleb, Ouadii Mouaqit

Department of Visceral Surgery, Faculty of Medicine and Pharmacy of Fez, Hassan II University Hospital Fez, Sidi Mohamed Ben Abdellah University, Fez, Morocco

DOI: $10.36347 /$ sasjs.2020.v06i07.005

| Received: 02.07.2020 | Accepted: 09.07.2020 | Published: 28.07.2020

*Corresponding author: Khalid Mazine

\section{Abstract}

Pancreatic cancer is the fifth leading cause of cancer deaths in Western countries. Despite advances in imaging, the resectability of carcinomas of the pancreas remains very low with rates ranging from 5 to $22 \%$. In fact, about $1 / 4$ of the tumors found to be resectable in the preoperative assessment appear unresectable at laparotomy. This poor prognosis is attributable to late stage presentation, lack of effective treatments, early recurrence and absence of clinically useful biomarkers which can detect pancreatic cancer in its precursor forms or earliest stages. Finding a sensitive, easy, lowrisk and low-cost tool for assessing resectability has been a sought-after goal for many years. We aimed to study the intrinsic validity characteristics of the CA 19-9 serum level for the evaluation of the resectability of pancreatic tumors, compared with surgical exploration data. We retrospectively reviewed, between 2014 and 2019, preoperative serum levels of CA19-9 in patients with pancreatic cancers diagnosed by imaging means. We calculated the sensitivity and specificity of the Carbohydrate antigen 19-9 (CA 19-9) to evaluate the resectability as opposed to surgical exploration. The performance of the serum CA 19-9 levels was compared with the surgical evaluation of the resectability according to receiver operating characteristic (ROC) curve analysis, in order to determine the optimum cut-off value for the CA 19-9 level preoperatively that will indicate that the pancreatic cancer is resectable. 96 patients were included. $34.3 \%$ were resectable $(\mathrm{N}=33)$, and $25 \%$ were judged operable and resectable $(\mathrm{N}=24)$. The mean CA 19-9 level was 250,5 $\mathrm{U} / \mathrm{mL}$ in the resectable group and $794,2 \mathrm{U} / \mathrm{mL}$ in the unresectable group. The threshold value found of CA19-9 serum levels to predict resectability of pancreatic cancers was $122 \mathrm{U} / \mathrm{mL}$ with a specificity and sensitivity of $97,2 \%$ and $81,8 \%$ respectively. The positive predictive value of CA19-9 serum levels for pancreatic cancer resectability was $94,7 \%$, whereas the negative predictive value was $89,7 \%$. Our study confirms the importance of CA19-9 serum levels in pancreatic cancers. This biomarker may be useful for determining preoperatively resectable patients (CA 19-9 $<122 \mathrm{U} / \mathrm{mL}$ ), as well as those requiring additional assessment, such as an exploratory laparoscopy (CA 19-9> 122U / $\mathrm{mL})$.

Keywords: Carbohydrate antigen 19-9 (CA 19-9), Pancreatic cancer, Resectability.

Copyright @ 2020: This is an open-access article distributed under the terms of the Creative Commons Attribution license which permits unrestricted use, distribution, and reproduction in any medium for non-commercial use (NonCommercial, or CC-BY-NC) provided the original author and source are credited.

\section{INTRODUCTION}

Treatment of carcinoma of the pancreas remains a challenge for clinicians; the overall prognosis of the disease remains dismal, with a 5-year actuarial survival rate of less than 5\% [1]. Surgical resection is the only chance for a cure, but the majority of patients have grossly unresectable disease at the time of diagnosis. In fact, only $20 \%$ of patients with pancreatic cancer will have a tumor that is considered surgically resectable at the time of diagnosis $[1,2]$. Although the goal of surgery is to remove the entire tumor with no residual disease (oncological R0 resection) [2]. the 5year survival rate following surgical resection of pancreatic cancer reported by leading medical centers does not exceed 25\% [ 3,4]. Curative resection of pancreatic adenocarcinoma can be performed in only $10 \%$ of the patients, and positive resection margin (microscopic, R1; or minimal macroscopic, R2) pancreatic tumors are associated with a poor prognosis $[5,6]$. Computed tomography (CT) is the established method for diagnosing and staging pancreatic cancer, although approximately $25 \%-30 \%$ of patients who are deemed to have resectable disease at CT ultimately have unresectable lesions at surgery [7]. This issue is important because recovery from the unnecessary laparotomy further delays palliative systemic therapy [8]. Developing new methods for early diagnosis and better modalities for a therapeutic intervention are our best hope for reducing the burden of pancreatic cancer. 
The 2 most studied tumor markers that have been evaluated in the diagnosis and prognosis of patients with pancreatic cancer are the Carcino-embryonic antigen (CEA) and carbohydrate antigen 19-9 (CA199). However, little is known about the association between the levels of these markers and the existence of metastasis or a locally advanced stage (LD) in patients with pancreatic cancer $[8,9]$. CA $19-9$ is a monosialoganglioside/glycolipid that can be detected in low levels in healthy individuals $(<40 \mathrm{U} / \mathrm{mL})$, and the level is elevated in several types of cancer, including pancreatic, hepatocellular, gastric, colorectal, and ovarian. Elevated CA19-9 levels can also be seen in benign conditions of extra hepatic biliary obstruction such as pancreatitis and choledocholithiasis [10]. This has limited the diagnostic utility of CA19-9 in patients who present with biliary obstruction of unclear cause. Several studies have shown that high levels of CA19-9 (>300 U/mL) correlate with advanced disease [11, 12]. However, most of these studies included patients with known metastatic disease.

Therefore, we tested the hypothesis that preoperative serum levels of CA19-9 are significantly elevated in patients with unresectable pancreatic cancer despite radiologic staging demonstrating resectable disease.

\section{PATIENTS AND METHODS}

The present study included 96 patients who had been treated surgically for potentially resectable primary pancreatic adenocarcinoma at the Department of Surgery, HASSAN II University Hospital, Fez, Morocco, from January 2014, through January 2019. We aimed to evaluate the resectability of pancreatic cancer using the CA 19-9 biomarker.

Pancreatic adenocarcinoma was histologically confirmed by pathologic examination of the resected specimen or, if unresected, by intraoperative biopsies; all other histologic variants, such as mucinous adenocarcinomas, intraductal papillary adenocarcinomas, acinar cell carcinomas, and endocrine tumors were excluded from the analysis.
The age of the patients ranged from 38 to 85 years (Mean age: 60 years), and 35 of them were women $(34 \%) .82$ pancreatic carcinomas involved the head, 8 the body, and 6 the tail. Patients had received no chemotherapy or radiation therapy prior to surgery. All patients' tumors were evaluated by preoperative boluscontrast computed tomography (CT) scan and were judged to be resectable if the tumor was limited to the pancreas without extension to the superior mesenteric artery, the coeliac trunk, the portal vein or the superior mesenteric vein and if there was no evidence of metastasis (liver, peritoneum or coeliac lymph nodes).

Laboratory results were reviewed for preoperative serum CA 19-9 levels in all 96 patients; we calculated the sensitivity and specificity of the CA 19-9 serum levels for the evaluation of resectability in correlation with surgical exploration. We used receiver operating curves (ROCs) and calculated the area under the curve (AUC) to assess the predictive characteristics of CA 19-9 serum levels with potential confounder variables. The closest value to the upper left corner of the graph was considered as a threshold value. We then proceeded to calculate the sensitivity, specificity, as well as the positive and negative predictive values of this threshold value. Statistical significance was assumed for $\mathrm{P}<0,05$. All statistical analyses were performed using SPSS ${ }^{\circledR}$.

\section{RESULTS}

After a multidisciplinary assessment of the 96 patients, 58(60\%) patients were operatively staged by laparotomy, 26 (25\%) were judged resectable, 22(23\%) underwent pancreatectomy

pancreaticoduodenectomy; 3 distal pancreatectomy) on the basis of tumor location. In 2 patients $(2,1 \%)$ a local artery invasion was found despite preoperative radiologic imaging demonstrating localized disease, they underwent surgical bypass, 2 patients were judged resectable but not operable (ASA IV) and underwent a surgical bypass, $32(33,5 \%)$ patients were found to have metastatic disease ( liver and/or peritoneum) or local invasion to the vessels and they underwent bypass surgery. 38 patients $(39,5 \%)$ underwent a non-surgical palliative treatment (Table 1).

Table-1: Baseline characteristics of patients with pancreatic adenocarcinoma

\begin{tabular}{|l|c|}
\hline \multicolumn{1}{|c|}{ Characteristics } & Finding \\
\hline Number of patients & 96 \\
\hline Sex, No. M/F & $61 / 35$ \\
\hline Age, y, mean \pm SD & $60 \pm 10,2$ \\
\hline Disease state, No. (\%) & $22(23 \%)$ \\
Localized & $72(75 \%)$ \\
Metastatic /Locally advanced & \\
\hline Treatment & $22(23 \%)$ \\
pancreatectomy & $36(37,5 \%)$ \\
surgical bypass & $38(40 \%)$ \\
non-surgical palliative treatment & \\
\hline
\end{tabular}


Most of the patients with a head location, had a biliary preoperative drainage, therefore $\mathrm{Ca} 19-9$ was obtained after the jaundice had resolved. The median CA19-9 level for the 58 operated patients was $588 \mathrm{U} / \mathrm{mL}$. For the patients with localized disease who underwent pancreatectomy, the median preoperative CA 19-9 level was $250 \mathrm{U} / \mathrm{mL}$. Patients with unresectable disease had a 3 -fold-higher preoperative serum level of CA19-9, with a median of $794 \mathrm{U} / \mathrm{mL}$. (Figure 1). For patients with unresectable tumors, if we distinguish locally advanced tumors from metastatic ones, it seems that the CA19-9 serum levels are correlated to the extension of the disease, the median level of CA19-9 for patients with locally advanced tumors was $770 \mathrm{U} / \mathrm{mL}$, whereas for metastatic tumors it was $887 \mathrm{U} / \mathrm{mL}$ (Table 2).

Table-2: Serum CA 19-9 levels of resectable, unresectable and metastatic pancreatic adenocarcinoma

\begin{tabular}{|c|cc|}
\hline Group & \multicolumn{2}{|c|}{ CA 19-9 U/mL } \\
& median & mean \\
\hline Resected $(n=22)$ & 70,16 & 250,5 \\
\hline localy advanced $(n=34)$ & 616,64 & 770,8 \\
\hline metastatics $(n=38)$ & 876,87 & 887,4 \\
\hline
\end{tabular}

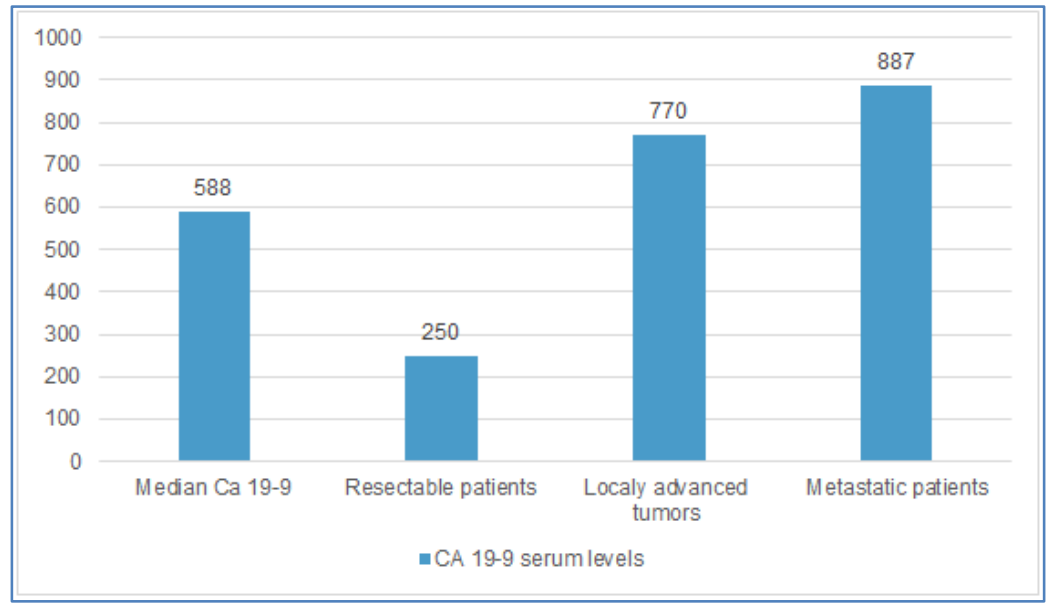

Fig-1: CA 19-9 serum levels

The area under the ROC curve was 0.823 ; the $95 \%$ confidence interval was 0.679 to 0.966 . This result suggested that changes in the CA 19-9 levels may be closely linked to resectability). When the cut-off value of CA 19-9 was accepted as $122 \mathrm{U} / \mathrm{mL}$, the specificity and sensitivity were $97,2 \% \%$ and $81.8 \%$ respectively (Table 3). On the other hand, preoperative resectability according to this cut-off point was compared to surgical staging and positive and negative predictive values of CA $19-9$ were $94,7 \%$ and $89,7 \%$ respectively (Table 4 ).

Table-3: AUC ROC of CA19-9 serum levels as predictive of pancreatic cancer resecability

\begin{tabular}{|c|l|l|l|l|c|}
\hline Variable & AUC ROC & \multicolumn{1}{c|}{$\boldsymbol{p}$} & Cut off level & Sensibility (\%) & Specificity (\%) \\
\hline CA 19-9 serum levels & 0,823 & $<0,001$ (significant) & 122 & 97,2 & 81,8 \\
\hline
\end{tabular}

Table-4: Positive and negative predictive values with cut off value of 122

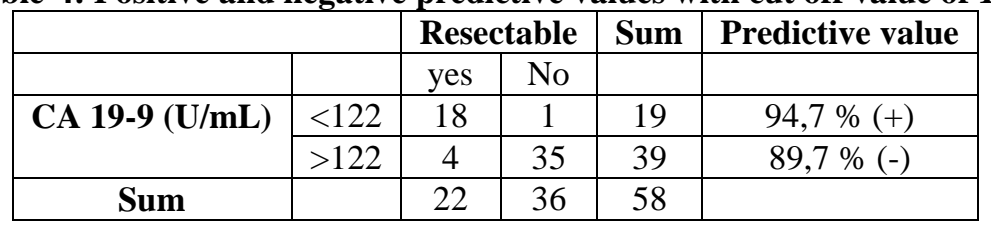

In the lights of these results, CA 19-9 serum level is a predictive factor of the resectability of pancreas tumors with a cut-off value of $122 \mathrm{U} / \mathrm{mL}$.

Figure 2 shows the correlation between serum levels of CA 19-9 and the operative findings in our cohort. Among the 39 patients with CA19-9 serum levels superior to $122 \mathrm{U} / \mathrm{mL}, 35(89,7 \%)$ had an unresectable tumor. The positive predictive value of CA 19-9 in the evaluation of the resectability is 94,7 $\%$. 


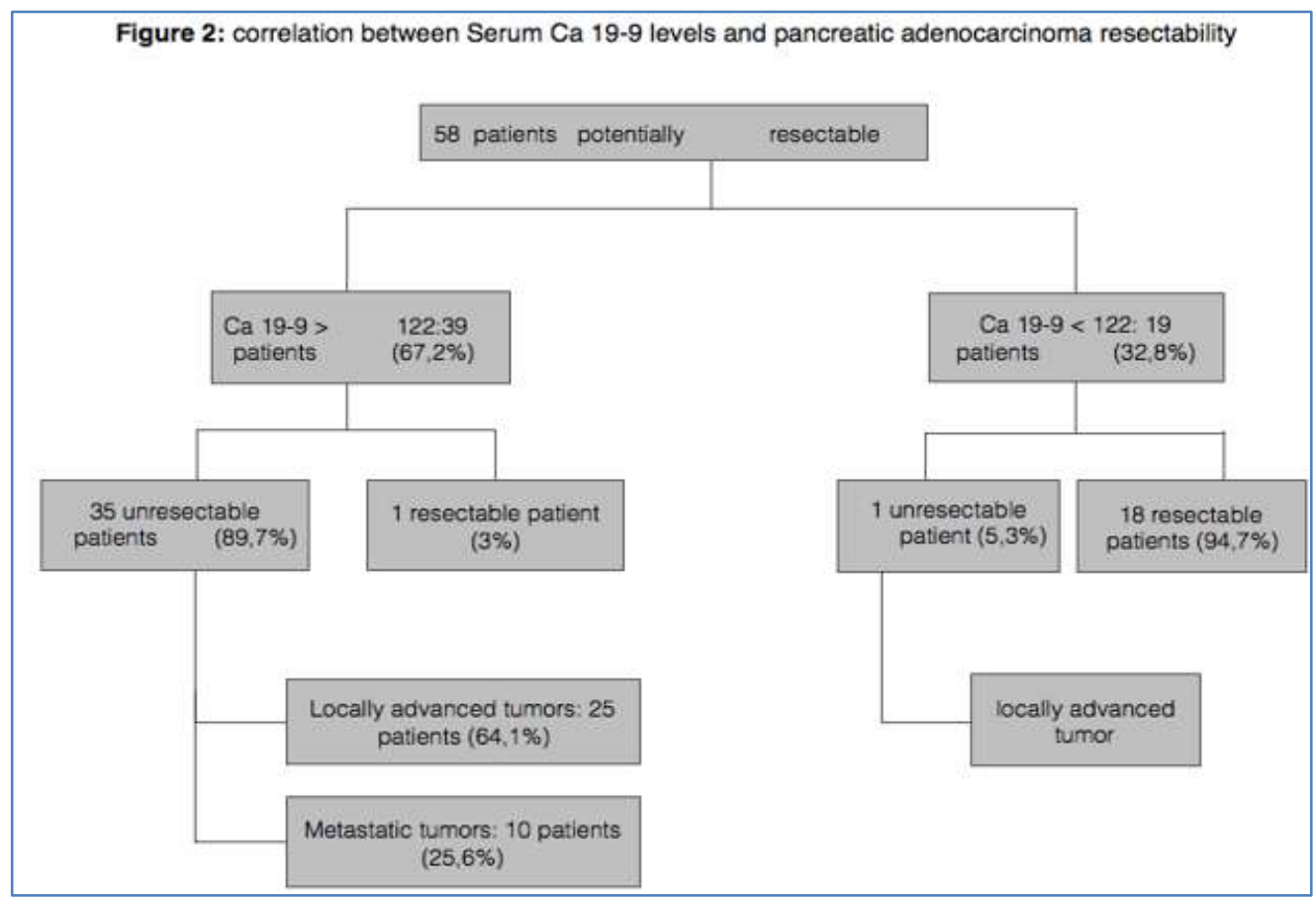

\section{DISCUSSION}

In recent years, the advances in diagnostic studies of pancreatic cancer have been remarkable, with modalities including dual-phase helical CT and laparoscopic ultra-sonography. Therefore, the accuracy of preoperative assessment of unresectability, distant metastases, and lymph node metastases of pancreatic cancer has improved significantly. Although technology for this imaging modality has tremendously improved during the last 2 decades, it still misses occult peritoneal or liver metastatic disease $(<1 \mathrm{~cm})$ in $4 \%$ to $15 \%$ and occult vascular involvement in $4 \%$ to $19 \%$ of the cases $[11,13,14]$. Other modalities of preoperative staging, such as positron emission tomographic scanning and laparoscopy, are being evaluated to improve the detection of unresectable disease. Even when state-ofthe-art imaging is available, a substantial number of patients are still found to have occult metastatic disease at the time of surgical exploration. Therefore, we hypothesized that excessively elevated preoperative serum levels of the tumor marker CA 19-9 can be used as an adjunct indicator of unresectable pancreatic adenocarcinoma in patients deemed to have localized disease on the basis of preoperative CT scan.

Since the first means of antibody detection for CA 19-9 were described in 1979 [15], it has been found to be the most useful tumor marker in the diagnosis, prognostic prediction, and evaluation of recurrent disease in pancreatic adenocarcinomas [16, 17]. In one of the first studies evaluating CA 19-9 as a prognostic indicator M Sternberg et al. [18] studied 37 patients with any stage of pancreatic adenocarcinoma and found that patients with metastatic disease had a higher mean CA 19-9 level $(1656 \mathrm{U} / \mathrm{mL})$ than those with resectable disease $(423,8 \mathrm{U} / \mathrm{mL})$. During the following 10 years, studies by Tian et al. [19], Van den Bosch et al. [12], and Safi et al. [20], demonstrated similar results. However, these studies included patients with radiologic evidence of metastatic disease and did not specifically analyze the group of patients with potentially resectable pancreatic cancer on the basis of preoperative imaging studies.

Forsmark et al. [21] Demonstrated that patients with CA 19-9 levels greater than $300 \mathrm{U} / \mathrm{mL}$ have advanced tumors, and resection is rarely possible in these cases. More recently, Schlieman and associates [22] demonstrated that among patients whose preoperative imaging studies indicated resectable pancreatic cancer, laparotomy may show that those with abnormally high serum levels of CA 19-9 may have unresectable disease. In their series of the 89 patients, 25 had locally advanced (unresectable) disease and 24 had metastatic disease. The mean adjusted CA 19-9 level was significantly lower in those with localized disease than those with locally advanced or metastatic disease. When a threshold-adjusted CA 19-9 level of $150 \mathrm{U} / \mathrm{mL}$ was used, the positive predictive value for unresectable disease was $88 \%$. On the other hand, in all these studies, arbitrary cut-off points were used. An efficient way to display and assess the predictive value of a parameter throughout a range of cut-off points is with ROC curves [23] which we used in our study.

An elevated CA19-9 level is not consistently observed for patients with metastatic disease for several reasons. First, patients who are negative for Lewis antigen (a-, b-) do not synthesize CA 19-9, and this constitutes $4 \%$ to $15 \%$ of the population $[19,17]$. We did not test for Lewis antigen status in our study. Second, hyperbilirubinemia caused by the malignant obstruction of the common bile duct elevates CA 19-9 
levels. This is believed to be due to hepatic insufficiency to degrade and secrete CA 19-9[17]. Several studies have shown that the association of elevated levels of CA19-9 with the diagnosis of pancreatic cancers is significantly obscured in the face of obstructive jaundice, and the cutoff value should be adjusted to hyperbilirubinemia [22, 24, 25]. Other studies didn't find a significant difference in bilirubin levels among groups of patients with or without obstructive jaundice [23]. We did not use adjusted CA19-9 levels to hyperbilirubinemia.

Our results are similar to those of the literature (Table 5). It is true that their threshold value is higher than ours; but this can be explained by the facts that we didn't use adjusted values of CA 19-9 to bilirubinemia and the difference of the number of patients included.

Table-5: Comparison of our result with some published studies

\begin{tabular}{|l|l|l|l|l|l|}
\hline Author, year & CA 19-9 cutoff value & sensitivity \% & specificity \% & PPV \% & NPV \% \\
\hline Schleiman et al. $2003[22]$ & 6 & - & 66 & 88 & 66 \\
\hline Kiliç et al. $2004[23]$ & $256,4 \mathrm{U} / \mathrm{mL}$ & 82,4 & 92,3 & 91,4 & 83,9 \\
\hline Zhang et al. $2008[28]$ & $353,15 \mathrm{U} / \mathrm{mL}$ & 91,3 & 78,3 & 84,38 & 90 \\
\hline Our study & $122 \mathrm{U} / \mathrm{mL}$ & 97,2 & 81,8 & 94,7 & 89,7 \\
\hline
\end{tabular}

The correlation between CA 19-9 serum levels and pancreatic cancer resectability is not universal, and is undermined by the facts that $5-10 \%$ of patients with pancreatic cancer will not demonstrate elevated CA 199 serum levels given their Lewis negative state, and by false positive elevations in obstructive jaundice. CA 199 serum levels alone should not be the sole criteria used in making decisions to proceed to surgery; rather the CA 19-9 serum level is one of several contributing factors used in combination with clinical evaluation and information obtained from radiological and endoscopic imaging [26, 27]. In our study, the pancreatic tumor was resectable in only four patients with CA19-9 serum levels > $122 \mathrm{U} / \mathrm{mL}$, this result suggests that a high CA 19-9 serum level $(>122 \mathrm{U} / \mathrm{mL})$ can be used as a selection criteria for patients in view of a diagnostic laparoscopy in order to detect unresectable tumors, particularly in cases where the preoperative analysis (CT scan) indicates a resectable tumor, and to avoid unnecessary laparotomies.

\section{CONCLUSION}

The CA 19-9 level may be a useful marker for determining preoperatively which patients have unre sectable pancreatic cancer. The presence of an elevated CA 19-9 level (>122U/mL) should direct the surgeon toward a more liberal use of staging laparoscopy, in order to avoid an unnecessary laparotomy.

\section{REFERENCES}

1. Fujioka S, Misawa T, Okamoto T, Gocho T, Futagawa Y, Ishida Y, Yanaga K. Preoperative serum carcinoembryonic antigen and carbohydrate antigen 19-9 levels for the evaluation of curability and resectability in patients with pancreatic adenocarcinoma. J Hepatobiliary Pancreat Surg. 2007; 14(6):539-544.

2. Beger HG, Gansauge F, Leder G. Pancreatic cancer: who benefits from curative resection? Can J Gastroenterol 2002 Feb;16(2):117-20.

3. Sener SF, Fremgen A, Menck HR, Winchester DP. Pancreatic cancer: a report of treatment and survival trends for 100313 patients diagnosed from 1985-1995, using the National Cancer Database. J Am Coll Surg. 1999 Jul;189(1):1-7

4. Pasquali C, Sperti C, Filipponi C, Pedrazzoli S. Epidemiology of pancreatic cancer in Northeastern Italy: incidence, resectability rate, hospital stay, costs and survival (1990-1992). Dig Liver Dis. 2002 Oct;34(10):723-31.

5. Neoptolemos JP, Stocken DD, Dunn JA, Almond J, Beger HG, Pederzoli P. Influence of resection margins on survival for patients with pancreatic cancer treated by adjuvant chemoradia- tion and/or chemotherapy in the ESPAC-1 randomized controlled trial. Ann Surg. 2001 Dec;234(6):75868.

6. Baulieux J, Delpero JR. Surgical treatment of pancreatic cancer: curative resections. Ann Chir.2000 Sep;125(7):609-17.

7. Prokesch RW, Chow LC, Beaulieu CF, NinoMurcia M, Mindelzun RE, Bammer R, Huang J, Jeffrey RB. Local staging of pancreatic carcinoma with multi-detector row CT: use of curved planar reformations - initial experience. Radiology. 2002 Dec;225(3):759-65

8. Schlieman MG, Ho HS, Bold RJ. Utility of tumor markers in determining resectability of pancreatic cancer Arch Surg. 2003 Sep;138(9):951-5; discussion 955-6.

9. Safi F, Schlosser W, Kolb G, Beger HG. Diagnostic value of CA19-9 in patients with pancreatic cancer and nonspecific gastrointestinal symptoms. J Gastrointest Surg. 1997 MarApr;1(2):106

10. Lamerz R. Role of tumor markers, cytogenetics. Ann Oncol. 1999;10 Suppl 4:145-9.

11. Steinberg WM, Gelfand R, Anderson KK, Glenn J, Kurtzman SH, Sindelar WF, Toskes PP. Comparison of the sensitivity and specificity of the CA19-9 and carcinoembryonic antigen assays in detecting cancer of the pancreas. Gastroenterology. 1986 Feb;90(2):343-9.

12. Van den Bosch RP, van Eijck CH, Mulder PG, Jeekel J. Serum CA19-9 determination in the 
management of pancreatic cancer. Hepatogastroenterology. 1996 May-Jun;43(9):710-713.

13. Holzman MD, Reintgen KL, Tyler DS, Pappas TN. The role of laparoscopy in the management of suspected pancreatic and periampullary malignancies. J Gastrointest Surg. 1997 MayJun;1(3):236-43; discussion 243-4.

14. Saldinger PF, Reilly M, Reynolds K. Is CT angiography sufficient for prediction of resectability of periampullary neoplasms? J Gastrointest Surg. 2000 May-Jun;4(3):233-7; 2389.

15. Koprowski H, Steplewski Z, Mitchell K, Herlyn M, Herlyn D, Futher P. Colorectal carcinoma antigens detected by hybridoma antibodies. Somat Cell Genet. 1979 Nov;5(6):957-71.

16. Riker A, Libutti SK, Bartlett DL. Advances in the early detection, diagnosis, and staging of pancreatic cancer. Sure Oncol. 1997 Nov;6(3):157-69.

17. Ritts RE, Pitt HA, CA 19-9 in pancreatic cancer. Surg Oncol Clin N Am. 1998 Jan;7(1):93-101.

18. Steinberg WM, Gelfand R, Andersn KK. Comparison of the sensitivity and specificity of the CA 19-9 and carcinoembryonic antigen assays in detecting cancer of the pancreas. Gastroenterology. 1986 Feb;90(2):343-9.

19. Tian F, Appert HE, Myles J, Howard JM. Prognostic value of serum CA 190-9 levels in pancreatic adenocarcinoma. Ann Surg. 1992 Apr;215(4):350-5.

20. Safi F, Schlosser W, Kolb G, Beger HG. Prognostic value of CA 19-9 serum course in pancreatic cancer. Hepatogastroenterology. 1998 Jan-Feb;45(19):253-9.

21. Forsmark CE, Lambiase L, Vogel SB. Diagnosis of pancreatic cancer and predic- tion of unresectability using the tumor associated antigen CA 19-9. Pancreas. 1994; 9:731-4.

22. Schlieman MG, Ho HS, Bold RJ. utility of tumor markers in determining resectability of pancreatic cancer. Arch Surg. 2003 sep; 138(9):951-5; discussion 955-6.

23. Kiliç M, Göçmen E, Tez M, Ertan T, Keskek M, Koç M. Value of preoperative serum CA 19-9 levels in predicting resectability for pancreatic cancer. Can J Surg; 49(4):241-244.

24. Mann DV, Edwards RS, Ho Siglazer G. Elevated tumor marker CA 19-9: clinical interpretation and influence of obstructive jaundice. Eur J Surg Oncol. 2000 Aug;26(5):474-9.

25. Mery CM, Durate-Rojo A, Paz-Pineda F, Gomez E, Robles-Diaz G. Does cholestasis change the clinical usefulness of CA19-9 in pancreatobiliary cancer? Rev Invest Clin. 2001 NovDec;53(6):511-7.

26. Ballehaninna UK, Chamberlain RS. The clinical utility of serum CA 19-9 in the diagnosis, prognosis and management of pancreatic adenocarcinoma: An evidence-based appraisal. J Gastrointest Oncol. 2012;3(2):105-119.

27. Zhang S, Wang YM, Sun CD, Lu Y, Wu LQ. Clinical value of serum CA19-9 levels in evaluating resectability of pancreatic carcinoma. World J Gastroenterol. 2008 Jun 21; 14(23): 3750-3753. 